NBER WORKING PAPER SERIES

\title{
OPTIMAL STABILIZATION RULES IN A STOCHASTIC MODEL OF INVESTMENT WITH GESTATION LAGS
}

John B. Taylor

Working Paper No. 1225

NATIONAL BUREAU OF ECONOMIC RESEARCH 1050 Massachusetts Avenue Cambridge, MA 02138

November 1983

The research reported here is part of the NBER's research program in Economic Fluctuations. Any opinions expressed are those of the author and not those of the National Bureau of Economic Research. 
NBER Working Paper \#1225

November 1983

Optimal Stabilization Rules in a Stochastic

Model of Investment with Gestation Lags

\begin{abstract}
This paper considers the problem of calculating optimal policy rules to stabilize fluctuations in investment in an economy where firms' investment behavior can be described by a dynamic optimization model. In the optimization model, the dynamics of investment are generated by heterogeneous gestation lags between the start and completion of capital projects, rather than by adjustment costs in the installation of capital. A procedure is derived for calculating policy rules for an arbitrary autoregressive process generating fluctuations in firms sales. Through stochastic simulation we investigate the effects of using certain suboptimal policy rules in cases where there are constraints against using the optimal rules.
\end{abstract}

John B. Taylor

Rm. 404 - Woodrow Wilson School

Princeton University

Princeton, NJ 08544 


\title{
Optimal Stabilization Rules in a Stochastic \\ Model of Investment with Gestation Lags*
}

\author{
by \\ John B. Taylor \\ - Princeton University
}

\section{Introduction}

In recent years there has been an extensive amount of economic research devoted to deriving investment demand functions from stochastic dynamic models of firm behavior. I/ Two advantages of such derived demand functions are related to economic policy and have motivated much of this research. First, the parameters of the demand functions depend explicitly on technological properties of the firm's production process and therefore can be assumed to be independent of economic policy which is external to the firm. Second, the investment demand functions show how

*The statistical time series methodology that is used to derive the optimal countercyclical stabilization rules in this paper was first taught to me by Ted Anderson in his time series and econometrics lectures at Stanford University in 1970 and 1971. I am indebted to him for his stimulating and clear presentations, and for his patience and personal dedication as a teacher and advisor in an area that has proved enormously useful in my research.

The research reported in this paper has been supported by a grant from the National Science Foundation at Princeton University and at the National Bureau of Economic Research. Useful programing assistance was provided by Jungyoll Yun. 
the firm's decisions depend on expected future variables, and thereby permit one to investigate how anticipations of future policy actions might influence the effectiveness of economic policy. Reduced-form functions in which investment demand is written as a fixed distributed lag of past variables, regardless of the stochastic process affecting these variables, do not have these advantages.

Although policy questions have been investigated using dynamic models of firm investment behavior, to date there has been little research on the calculation or characterization of optimal policy using such models. $2 /$ In this paper we consider the problem of finding optimal control ruies to stabilize fluctuations in investment demand using such a model. In the model used here the dynamics of investment are generated by heterogeneous gestation lags between the start and completion of capital projects, rather than by adjustment costs in the installation of capital. Gestation lags permit an analytic calculation of optimal stabilization policy under a wide range of stochastic processes generating firms' desired capital stock, and potentially can be estimated using technological data on capital construction.

The paper is organized as follows. In Secticn 2 the dynamic investment model is presented and an investment demand equation is derived. In Section 3 a procedure for calculating the optimal stabilization policy rules is derived for an arbitrary autoregressive process generating the fluctuations in sales. In Section 4 the optimal rules are calculated for the case of a second-order autoregressive business cycle model. In Section 5 we examine through stochastic simulation the effects of using certain suboptimal policy rules.which might be employed when there are practical constraints on the design of the optimal rules. 
2. An investment model with heterogeneous gestation lags $\underline{3}$ /

Suppose that firms use $n$ different types of capital inputs. Let the stock of capital of type $i$ at the start of time period $t$ be denoted by $k_{i t}, i=1, \ldots, n$. The types of capital differ in their gestation times; that is the time it takes to build a unit of capital. Capital of type $i$ is assumed to take $i$ periods to build. Let $s_{i t}$ be the value of capital projects of type $i$ started at time $t$. Then we have

$$
k_{i t+i}=\left(1-h_{i}\right) k_{i t+i-1}+s_{i t}
$$

where $b_{i}$ is a constant proportional depreciation rate for each type of capital. According to equation (1) capital projects of type $i$ started at time $t$ are completed and added to the capital stock at time $t+i$. Depreciation of the amount $h_{i} k_{i t+i-1}$ is subtracted from gross completions to get the net increase in capital. 4 /

Investment expenditure, or "value put in place," during the gestation period of each project depends on the technology of construction. Let $x_{i t}$ be the value put in place on a capital project of type $i$ during period $t$. Let $w_{i j}$ be the fraction of the project of type $i$ put in place during the $j^{\text {th }}$ period following the start of the project. Then total investment expenditures on projects of type $i$ are given by the distributed lag

$$
x_{i t}=\sum_{j=1}^{i} w_{i j} s_{i t-j+1}
$$

for $1=1, \ldots, n$. Note that $\sum_{j=1}^{i} w_{i j}=1$ for each $1=1, \ldots, n$ and in particular that $w_{11}=1$. The fractions $w_{i j}$ are determined by the construction technology. In some cases such weights can be obtained in surveys. 
In order to obtain an investment demand function we assume that firms decide at each time period $\tau$ on a sequence of capital projects of each type in order to minimize the expected value of the intertemporal objective

$$
\sum_{t=\tau}^{\infty} \beta^{t}\left[.5 \sum_{i=1}^{n} d_{i}\left(v_{i} y_{t}-k_{i t}\right)^{2}+\sum_{i=1}^{n} c_{i t} x_{i t}\right],
$$

where $\beta$ is a discount factor, $v_{i}$ and $d_{i}, i=1, \ldots, n$ are fixed positive parameters, the $c_{i t}$ are the costs of investment goods of type $i$, and $y_{t}$ is a measure of sales. The variable $y_{t}$ is assumed to follow a known univariate stochastic process exogenous to the firm. As will be explained below the variables $c_{i t}$, which are also exogenous to the firm, will be policy determined as a function of $y_{t^{*}}$. The interpretation of (3) is that a firm's production process calls for capital of each type in a fixed ratio $v_{i}$ to total sales $y_{t}$, and that it is costly for the firm to deviate from that amount of capital in either a positive or a negative direction. This approach is similar to assuming a fixed coefficient production function with capital input coefficients equal to $\mathrm{v}_{i}^{-1}$, but it permits more flexibility in that the firm can deviate (at some cost) from these input coefficients. Note that we assume that there are no interaction affects in the costs of deviating from these input coeficients for different types of capital: one type of capital deviating from its appropriate level, neither increases nor decreases the costs of another type of capital deviating from its appropriate level. The lack of interaction makes possible a convenient analytical solution of the model, and seems reasonable given the fixed coefficient production interpretation of the objective function. 
By substituting equation (1) and (2) into (3) and differentiating with respect to the $k_{i t}$, noting that $k_{i t+i}$ or equivalently $s_{i t}$ is a decision variable at time $t$, the following optimal level of starts can be obtained for each time period

$$
\begin{aligned}
s_{i t}=v_{i} \hat{y}_{t+i} & -\left(1-h_{i}\right) k_{t+i-1} \\
& -\frac{1}{\beta^{i} d_{i}} \sum_{j=0}^{i-1} \beta^{j} w_{i j+1}\left(\hat{c}_{i t+j}-\beta\left(1-h_{i}\right) \hat{c}_{i t+j+1}\right),
\end{aligned}
$$

where the hat over a variable represents its minimum mean square predictor, or conditional expectation given information through period $t$. In the case of $y_{t}$, for example, $\hat{y}_{t+i}=E\left(y_{t+i} \mid y_{t}, y_{t-1}, \ldots\right)$. Equation (4) holds for each type of project from $i=1, \ldots, n$ and can be substituted into (2) in order to obtain the demand for investment. Note that equation (4) indicates that the resulting investment demand function depends explicitly on technological parameters and on expectations of future variables, a general property of demand functions obtained from intertemporal investment models mentioned in the introduction.

In the special case where the depreciation rates $h_{i}=0$ and the discount factor is equal to 1 , the optimal level of starts depends on a distributed lead in the expected changes in the cost of investment goods. In the case where depreciation rates are $h_{i}=1$, the distributed lead is in the level of the costs of investment goods.

\section{Optimal Policy Rules}

The model has been designed so that $y_{t}$ is a correlated disturbance that causes fluctuations in investment. We view $y_{t}$ as driven by an 
exogenous time series process representing, for example, business cycle fluctuations. One objective of policy is to reduce the fluctuations in investment by using investment incentives to offset the influence of this disturbance. Investment incentives affect the actual cost paid by firms for investment goods which we have represented by $c_{i t}$ in the model. Hence, the optimal control problem we consider is that of choosing a sequence of policy instruments $c_{i t}$ so as to minimize the fluctuations in the target $x_{i t}$. The optimal choice of $c_{i t}$ depends on the stochastic process for $y_{t}$. As with most optimal control or regulator problems the effect of the disturbances can be completely offset if there are a sufficient number of instruments. As indicated by (4), the number of instruments needed for complete offset is equal the number of different types of capital. In principle, therefore, it is necessary to have investment incentives for each type of capital so that each of the $c_{i t}$ can be set independently. In practice, tax incentives have differed for capital with different useful lives, but not for capital with different gestation periods. $\underline{5}$ /

In order to offset the effects of demand fluctuations on investment it is necessary that the cost variable $c_{i t}$ respond to $y_{t}$ in such a way that the forecasts of future values of $c_{i t}$ exactly offset the forecasts of future $y_{t}$ in equation (4). That is, $c_{i t}$ needs to be set so that

$$
\beta^{i} d_{i} v_{i} \hat{y}_{t+i}=\sum_{j=0}^{i-1} \beta^{j} w_{i j+1}\left(\hat{c}_{i t+j}-\beta\left(1-h_{i}\right) \hat{c}_{i t+j+1}\right) .
$$

for $i=1, \ldots, n$. It is clear from equation (4) that such a choice of $c_{i t}$ will eliminate the effect of the distrubance $y_{t}$ on starts and thereby on investment expenditures. Our objective is to calculate and characterize 
these optimal $c_{i t}$.

Assume that $y_{t}$ is determined by the following $p^{\text {th }}$ order autoregressive process.

$$
y_{t}=\alpha_{1} y_{t-1}+\ldots+\alpha_{p} y_{t-p}+u_{t}
$$

where $u_{t}$ is an uncorrelated random variable with a zero mean. Equation (6) can be used to generate predictions of the future values of $y_{t}$ that appear in equation (5) using results from prediction theory. See Anderson (1971, ch. 5). In order to obtain the optimal rule for the determination of the $c_{i t}$ we start with the general linear form

$$
c_{i t}=g_{i 1} y_{t}+g_{i 2} y_{t-1}+\ldots+g_{i p} y_{t-p+1}
$$

where the coefficients $g_{i l}$ through $g_{i p}$ are as yet undetermined. Predictions of future $c_{i t}$ can be obtained using (7) and the predictions of $y_{t}$ generated by (6). The problem of finding the optimal rule is thus reduced to the problem of finding the values of the coefficients that satisfy equation (5) for all $t$. These values can be found by substituting into (5) the forecasts of $y_{t}$ and $c_{i t}$ using (6) and (7), and finding the values of $g_{i l}$ through $g_{i p}$ which bring the coefficients of $y_{t}$ through $y_{t-p+1}$ to equality on both sides of (5). We now show how this procedure results in a set of linear equations in $g_{i l}$ through $g_{i p}$ which are straightforward to solve, even for fairly large values of $n$ and $p$. The procedure has some similarities to the feedforward control schemes proposed by Box and Jenkins (1970, ch. 12) for conventional linear regulator problems. 
The forecasts of future $y_{t}$ are given by

$$
\hat{y}_{t+s}=\gamma_{s 1} y_{t}+\gamma_{s 2} y_{t-1}+\ldots+\gamma_{s p} y_{t-p+1} \text {, for } s \geq 1 \text {, }
$$

where the $\gamma$-coefficients can be obtained recursively from the equations

$$
\gamma_{s j}=\alpha_{j} \gamma_{s-1,1}+\gamma_{s-1, j+1}, \quad j=1, \ldots, p-1,
$$

$$
\gamma_{s p}=\alpha_{p} \gamma_{s-1,1}
$$

The recursion starts at $s=1$ with $\gamma_{1 j}=\alpha_{j}, j=1, \ldots, p$. See Anderson (1971, p. 168) for a derivation of the recursion relationships in (9). Note also that $\hat{y}_{t+s}=y_{t+s}$ for $s<1$. The forecasts of future $c_{i t}$ are

$$
\hat{c}_{i t+s}=\sum_{j=1}^{s} g_{i j} \hat{y}_{t+s-j+1}+\sum_{j=s+1}^{p} g_{i j} y_{t+s-j+1},
$$

where the values for $\hat{y}_{t+s-j+1}$ can be obtained from (8).

Starting with the case where $i=1$ (the single period construction projects) we substitute these forecasting equations into (5) as follows. When $i=1$ equation (5) becomes:

$$
\beta d_{1} v_{1} \hat{y}_{t+1}=w_{11}\left(\hat{c}_{1 t}-\beta\left(1-h_{1}\right) \hat{c}_{1 t+1}\right),
$$

which can be written as

$$
\begin{aligned}
B d_{1} v_{1} \hat{y}_{t+1}=w_{11}\left(g_{11} y_{t}+\ldots+g_{1 p} y_{t-p+1}\right) \\
\quad-B\left(1-h_{1}\right)\left(g_{11} \hat{y}_{t+1}+g_{12} y_{t}+\ldots+g_{1 p} y_{t-p+2}\right),
\end{aligned}
$$


after substitution of $\hat{c}_{i t}$ and $\hat{c}_{1 t+1}$ from (10) with $s=1$ and $i=1$. Using equation (8) to substitute for $\hat{y}_{t+1}$ in (12), we obtain

$$
\begin{aligned}
3 d_{1} v_{1}\left(\gamma_{11} y_{t}+\ldots+\gamma_{1 p} y_{t-p+1}\right) & =w_{11}\left(g_{11} y_{t}+\ldots+g_{i p} y_{t-p+1}\right) \\
& -\beta\left(1-h_{1}\right)\left[g_{11}\left(\gamma_{11} y_{t}+\ldots+\gamma_{1 p} y_{t-p+1}\right)\right. \\
& \left.+g_{12} y_{t}+\ldots+g_{1 p} y_{t-p+2}\right] w_{11} .
\end{aligned}
$$

Equating the coefficients of $y_{t}, y_{t-1, \ldots, y_{t-p+1}}$ in (13) results in a set of linear equations in $g_{11}$ through $g_{i p}$ which will be useful to write out in detail

$$
\begin{aligned}
& \beta d_{1} v_{1} \gamma_{11}=w_{11}\left(1-\beta\left(1-h_{1}\right) \gamma_{11}\right) g_{11}-w_{11} \beta\left(1-h_{1}\right) g_{12}, \\
& \beta d_{1} v_{1} \gamma_{12}=-w_{11} \beta\left(1-h_{1}\right) \gamma_{12} g_{11}+w_{11} g_{12}-w_{11} \beta\left(1-h_{1}\right) g_{13}, \\
& \beta d_{1} v_{1} \gamma_{13}=-w_{11} \beta\left(1-h_{1}\right) \gamma_{13} g_{11}+w_{11} g_{13}-w_{11} \beta\left(1-h_{1}\right) g_{14},
\end{aligned}
$$

$$
\begin{aligned}
\dot{\cdot} & \\
\beta d_{1} v_{1} \gamma_{1 p-1} & =-w_{11} \beta\left(1-h_{1}\right) \gamma_{1 p-1} g_{11}+w_{11} g_{1 p-1}-w_{11} \beta\left(1-h_{1}\right) g_{1 p}, \\
B d_{1} v_{1} \gamma_{1 p} & =-w_{11} \beta\left(1-h_{1}\right) \gamma_{1 p} g_{11}+w_{11} g_{1 p} .
\end{aligned}
$$

Although we have written (14) using the general notation introduced for an arbitrary gestation $1 \mathrm{ag}$, in this case we have that $\gamma_{i j}=\alpha_{j}, j=1, \ldots, p$ and $w_{11}=1$. The $p$ equations in (14) are clearly linear in the $\mathrm{p}$ unknowns $g_{11}$ through $g_{1 p}$ and can be solved to obtain the optimal control rule for $c_{1 t}$. In the special case of full depreciation (h=1) the off-diagonal 
terms in the system of equations in (14) are equal to zero, so that the solution is given simply by $g_{1 j}=B d_{1} v_{1} \alpha_{j}$ for $j=1, \ldots, p$. In this special case the optimal control coefficients are proportional to the coefficients of the difference equation generating the disturbance $y_{t}$. The equations in (14) can alternatively be organized in matrix form. Letting $g_{i}=\left(g_{i 1}, \ldots, g_{i p}\right)^{\prime}$ and $Y_{s}=\left(\gamma_{s .1}, \ldots, \gamma_{s p}\right)^{\prime}$ the equation system becomes

$$
A_{1} g_{1}=\gamma_{1} \beta d_{1} v_{1}
$$

where $A_{1}$ is a $\mathrm{p} x \mathrm{p}$ matrix. Denoting the representative element of $\mathrm{A}_{1}$ by $a_{j m}^{(1)}$ the non-zero elements of the matrix are given by

$$
\begin{array}{ll}
a_{11}^{(1)} & =w_{11}\left(1-\beta\left(1-h_{1}\right) \gamma_{11}\right), \\
a_{j j}^{(1)}=w_{11}, & j=2, \ldots, p,
\end{array}
$$

$$
\begin{array}{ll}
a_{j-1, j}^{(1)}=-w_{11} \beta\left(1-h_{1}\right), & j=2, \ldots, p, \\
a_{j 1}^{(1)}=-w_{11} \beta\left(1-h_{1}\right) \gamma_{1 j}, & j=2, \ldots, p,
\end{array}
$$

and all other elements are equal to zero. The optimal values for the control rule coefficients for $c_{1 t}$ are then written as

$$
g_{1}=\stackrel{A}{-1}_{1}^{-1} \Upsilon_{1} \beta d_{1} v_{1}
$$

This same procedure can be used to compute the control rule coefficients for the $c_{\text {it }}$ variables corresponding to the longer gestation lags. That is, the forecasting equations with values of $i$ from 2 through 
n can be substituted into (5), and equations in the control rule coefficients can be obtained by equating coefficients of $y_{t}, y_{t-1}, \ldots$, $y_{t-p+1}$. For each value of $i$ there will be $p$ linear equations in $p$ unknowns. Before considering the results for the general case it is useful to consider the equations for $i=2$. In this two-period case

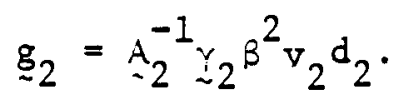

The non-zero elements of $\mathrm{A}_{2}$ are given by

$$
\begin{aligned}
& a_{11}^{(2)}=w_{21}+\beta\left(w_{22}-\left(1-h_{2}\right) w_{21}\right) \gamma_{11}-\beta^{2} w_{22}\left(1-h_{2}\right) \gamma_{21} \\
& a_{j 1}^{(2)}=\beta\left(w_{22}-\left(1-h_{2}\right) w_{21}\right) \gamma_{1 j}-\beta^{2} w_{22}\left(1-h_{2}\right) \gamma_{2 j}, \\
& a_{12}^{(2)}=\beta\left(w_{22}-\left(1-h_{2}\right) w_{21}\right)-\beta^{2} w_{22}\left(1-h_{2}\right) \gamma_{11}, \\
& a_{22}^{(2)}=w_{21}-\beta^{2} w_{22}\left(1-h_{2}\right) \gamma_{12},
\end{aligned}
$$

$$
\begin{array}{ll}
a_{j 2}^{(2)}=-\beta^{2} w_{22}\left(1-h_{2}\right) \gamma_{1 j}, & j=3, \ldots, p, \\
a_{j j}^{(2)}=w_{21}, & j=3, \ldots, p, \\
a_{j-1, j}^{(2)}=\beta\left(w_{22}-\left(1-h_{2}\right) w_{21}\right), & j=3, \ldots, p, \\
a_{j-2, j}^{(2)}=-\beta^{2} w_{22}\left(1-h_{2}\right), & j=3, \ldots, p .
\end{array}
$$

The remaining elements of $\mathrm{A}_{2}$ are equal to zero. Note that with full depreciation $\left(h_{2}=1\right)$ the matrix $A_{2}$ does not become diagonal, unlike in the one period projects. The development of the coefficients of $\mathrm{A}_{i}$ as $i$ increases from 1 to 2 , continues for $i$ equal 3 and so on, establishing a general formula which can be used for any value of $i$. 
In order to express the solution for $g$ in the general case, some additional notation is useful. Define a sequence $b_{i j}$

$$
\begin{aligned}
& b_{i 0}=w_{i 1} \\
& \left.b_{i j}=\beta^{j}\left(w_{i, j+1}-\left(1-h_{i}\right) w_{i, j}\right), \quad j=1, \ldots, i-1 \quad \text { (for } i \geq 2\right) \\
& b_{i i}=-\beta^{i}\left(1-h_{i}\right) w_{i i}
\end{aligned}
$$

for each $i=1, \ldots, n$. The $b_{i j}$ coefficients thus depend on the structural parameters of the model and are easily computed.

The solution in the general case can be written

$$
g_{i}=\underset{\sim i}{A} \gamma_{i}^{-I} \beta^{i} v_{i} d_{i}
$$

where the non-zero elements of the pxp matrix $\underset{\sim}{A_{i}}$, denoted by $a_{j m}^{(i)}$, are given by the following set of equations for $i=1, \ldots, n$,

$$
\begin{aligned}
& a_{j m}^{(i)}=b_{i, m-i}+\sum_{q=m}^{i} b_{i q} \gamma_{q-m+1, j}, \quad j=1, \ldots, m, \quad m=1, \ldots, i, \\
& a_{j m}^{(i)}=\sum_{q=m}^{i} b_{i q} \gamma_{q-m+1, j}, \quad j=m+1, \ldots, p, \\
& a_{j-i+r, j}^{(i)}=b_{i, i-r}, \quad \quad r=0, \ldots, i, \quad j=1, \ldots, i, \ldots, p .
\end{aligned}
$$

Note the equations in (22) are equivalent to the equation in (16) for $i=1$, and to the equations in (19) for $i=2$. These equations provide an easily computable way to evaluate the matrix $\underset{\sim i}{A_{i}}$ for an arbitrary $i$ and $p$. Hence, the entire set of optimal control coefficients ${\underset{i}{i}}_{i} i=1, \ldots, n$ can be computed. Since the dimension of the matrix $\underset{\sim i}{A}$ is equal to the order of the autogressive model generating the disturbances (which will usually be relatively small) and is not influenced by the length of the gestation lag (which could be quite long), computation costs should be low for this procedure. 
4. Properties of Optimal Policy in a Second Order Cyclical Model

In this section we examine the properties of the optimal rules for the case where sales disturbances $y_{t}$ follow a second order process $(p=2)$.

A second order model permits a fairly close approximation to the stochastic properties of business cycles observed in most countries, if $y_{t}$ is interpreted as proportional to detrended fluctuations in real GNP or some other measure of the state of aggregate economic activity.

For the second order model the optimal policy rules have the form

$$
c_{i t}=g_{i 1} y_{t}+g_{i 2} y_{t-1}, \quad i=1, \ldots, n,
$$

which is a special case of equation ( 7$)$. The control coefficients $g_{i l}$ and $g_{i 2}$ completely characterize the policy and of course are different for each type of capital i.

The policy coeficients associated with $i=1$, the single period projects, are obtained by solving equation (15) and are given by

$$
\begin{aligned}
& \left.g_{11}=\beta v_{1} \alpha_{1}\left[\frac{\alpha_{1}+\alpha_{2}\left(1-h_{1}\right)}{1-\beta\left(1-h_{1}\right)\left(\alpha_{1}+\alpha_{2}\left(1-h_{1}\right) \beta\right.}\right)\right] \\
& \left.g_{12}=\beta v_{1} d_{1}\left[\frac{\alpha_{2}}{1-\beta\left(1-h_{1}\right)\left(\alpha_{1}+\alpha_{2}\left(1-h_{1}\right) \beta\right.}\right)\right] \text {. }
\end{aligned}
$$

If depreciation occurs in one period $\left(h_{1}=1\right)$ then the policy rules can be characterized easily. In that case the policy reaction coefficients are proportional to the parameters of the autoregressive process $\alpha_{1}$ and $\alpha_{2}$. For example if $y_{t}$ is proportional to real GNP and $\alpha_{1}=1.4$ and $\alpha_{2}=$ -.5 , then the stabilization rules call for an increase in investment 
costs if real GNP is above normal levels, or if real GNP has been growing. For parameter values $\beta=1$ and $v_{1} d_{1}=1,(24)$ and (25) imply

$$
\begin{aligned}
c_{1 t} & =1.4 y_{t}+.5 y_{t-1} \\
& =.9 y_{t}+.5\left(y_{t}^{-y_{t-1}}\right) .
\end{aligned}
$$

Note that it is never optimal to react only to current $y_{t}$ unless $\alpha_{2}=0$, in which case the model is first-order model. As we show in the next section failure to react to lagged $y_{t}$ as in (26) can lead to a policy rule which destabilizes output. According to equation (26) investment costs should be raised by an extra amount if the real GNP has been growing.

The results are different if depreciation rates are smaller. The proportionality of the $g_{1 i}$ and $a_{i}$ will no longer hold, and the size of the reaction coefficients will be larger. Consider, for example the opposite extreme where $h=\dot{0}$. The stabilization rule becomes

$$
c_{1 t}=4 y_{t}+5\left(y_{t}-y_{t-1}\right)
$$

The reaction coefficients are much larger than in (26) and the size of the coefficient on the first difference of $y_{t}$ is larger relative to the size of the coefficient on the level of $y_{t}$.

\section{Stochastic Simulation Results with Suboptimal Policies}

The optimal policy rules derived and examined in the previous two sections have several features which are not usually characteristic of investment stabilization policy in practice. First, the policy is dynamic: 
lagged values of $y_{t}$ influence the optimal policy. In practice only the current level of $y_{t}$ seems to have been a factor in the determination of investment stabilization policy. Second, the policy instruments vary continuously with the values of $y_{t}$. In practice the policy instruments are likely to be set discretely - they are either on or off depending on the state of the business cycle. Third, the policy instrument must be targetted at the components of investment, distinguishing between different types of capital by gestation time. If the instrument is not targetted to each type of capital, perhaps because of the restriction that $c_{i t}=c_{j t}$ for $i \neq j$, then there will be an insufficient number of instruments and a constrained optimation approach is necessary. The methods developed in Chow (1980) might be used in such a situation. In this section of the paper we examine through the use of some simulation experiments what happens when policy is restricted to be suboptimal either because lagged values are omitted or because the instrument settings are limited to discrete values.

\section{Omission of Lagged Variables}

Consider the case where $n=1$ and $p=2$, and it is therefore optimal for $g_{12}$ to be nonzero. Suppose, however, that $g_{12}$ is restricted to be zero. In order to determine the possible impact of such a restricted investment policy on the stability of investment, we performed stochastic simulation for the set of parameter values for the intertemporal model calculated in Taylor (1982). These values are $v_{1}=.2, \mathrm{~d}_{1}=.07, \mathrm{~h}_{1}=.026$ and $\beta=.94$. We also set $\alpha_{1}=1.4$ and $\alpha_{2}=-.5$ as in the previous section. The variance of investment was then calculated by performing 1000 Monte Carlo simulations of 30 periods each, with the shocks $u_{t}$ being drawn from a normal distribution with mean 0 and variance $I$ and with the path of investment being determined by the model. The simulations 
were started from $k_{1,0}=0$. The variance of investment was found to be an increasing function of $g_{11}$ for this set of autoregressive parameter values. In the steady state (approximated at $t=30$ ), the variance of $x_{1 t}$ was equal to .00033 when $g_{11}=0$, increased to .00047 at $g_{11}=.002$, and increased further to .00076 when $g_{11}=.004$. Hence, this type of suboptimal policy could actually lead to perverse destabilization of investment. $-6 /$ This particular suboptimal policy is worse than no policy at all. Note that for this example the optimal values for $g_{11}$ and $g_{12}$ are .090 and -.048 respectively.

\section{Discrete Values for the Instruments}

Consider the case where $n=1$ and $p=1$. The optimal policy rule then has the form $c_{1 t}=g_{11} y_{t}$. Suppose, however, that only discrete changes in $c_{1 t}$ are feasible in practice, and that $c_{1 t}$ is therefore set according to the rule

$$
c_{t}= \begin{cases}c^{*} & \text { if } y_{t}>0 \\ 0 & \text { if } y_{t}=0 \\ -c^{*} & \text { if } y_{t}<0\end{cases}
$$

For this policy the forecasts of investment costs are not linear functions of $y_{t}$ as with the forecasting rules used in Section 2. Nevertheless the forecast of $c_{1 t+1}$ conditional on $y_{t}$, which is necessary for evaluating the decision rule (4), can be eyaluated for the case where $u_{t}$ is normally distributed. Using this conditional expectation for $\hat{c}_{1 t+1}$ and the rule in (28) we stochastically simulated the model with the same parameter values used for the previously described set of stochastic simulations. 
The results are shown in the first column of Table 1. (The other columns in Table 1 marked by the parameter $j$ signify a different discrete policy rule described below). The results indicate that while there is some reduction in the variance of investment with the discrete model, it is very small. Moreover when the step size (c*) increases beyond some small value the variance of investment begins to increase rapidly, indicating the potential for some destabilization. The restriction of $c_{1 t}$ to a discrete set of values results in a serious deterioration of the performance of the policy.

One of the reasons for the poor results with this suboptimal policy is that $c_{1 t}$ moves by a large amount when $y_{t}$ deviates only slightly from 0 . An improvement would therefore be expected if the rule were modified so that

$$
c_{1 t}= \begin{cases}c^{*} & \text { if } y_{t}>\hat{o} \\ 0 & \text { if }\left|y_{t}\right|<j \\ -c^{*} & \text { if } y_{t}<-j\end{cases}
$$

With rule (29) small movements in $y_{t}$ will not trigger a large response in $c_{1 t}$. Clearly equation (29) reduces to equation (28) when $j=0$. The simulation results for this alternative are shown in Table 1 in the columns marked with different values of $\delta$. As expected there is some reduction in the variance of $x_{\text {It }}$ but not as much as would be possible with the completely continuous optimal rule. Note also that Table 1 suggests that the best policy of the form (29) has $\delta$ between .2 and .3 and $c^{*}$ near .002 . These values depend on the parameters used in 
the simulation experiment, but they indicate the advantages of choosing the step-size and trigger points optimally even if policy is restricted to a discrete set of values. To the extent that such constraints are important in practice, further research to characterize how the best step-size and trigger values depend on the parameters of the model in this and more complicated examples would be useful.

\section{Concluding Remarks}

This paper has considered the problem of obtaining optimal control rules for stabilizing investment fluctuations in a model where investment demand depends on expected future values of the policy instruments. Simple expressions for evaluating the control rules were derived using results from prediction theory. These expressions were used to characterize some of the main properties of the control rules. In addition, the loss from using certain suboptimal rules was investigated. While suboptimal rules are clearly inferior to optimal rules, and in some cases inferior to no feedback rule at all, practical constraints on economic policy could lead to the use of such rules.

Although the formula for the control rule was derived for a particular dynamic investment model, the prediction theory approach that was employed could be used in other similar problems. The essential characteristic of the control problem studied here is that the target variable depends on forecasts of future values of the control instruments and on future exogenous variables. In the traditional control problem, the target variables depend on current and lagged values of the control instruments and the exogenous variables. This difference indicates why prediction theory is particularly useful for the type of problem studied in this paper. 


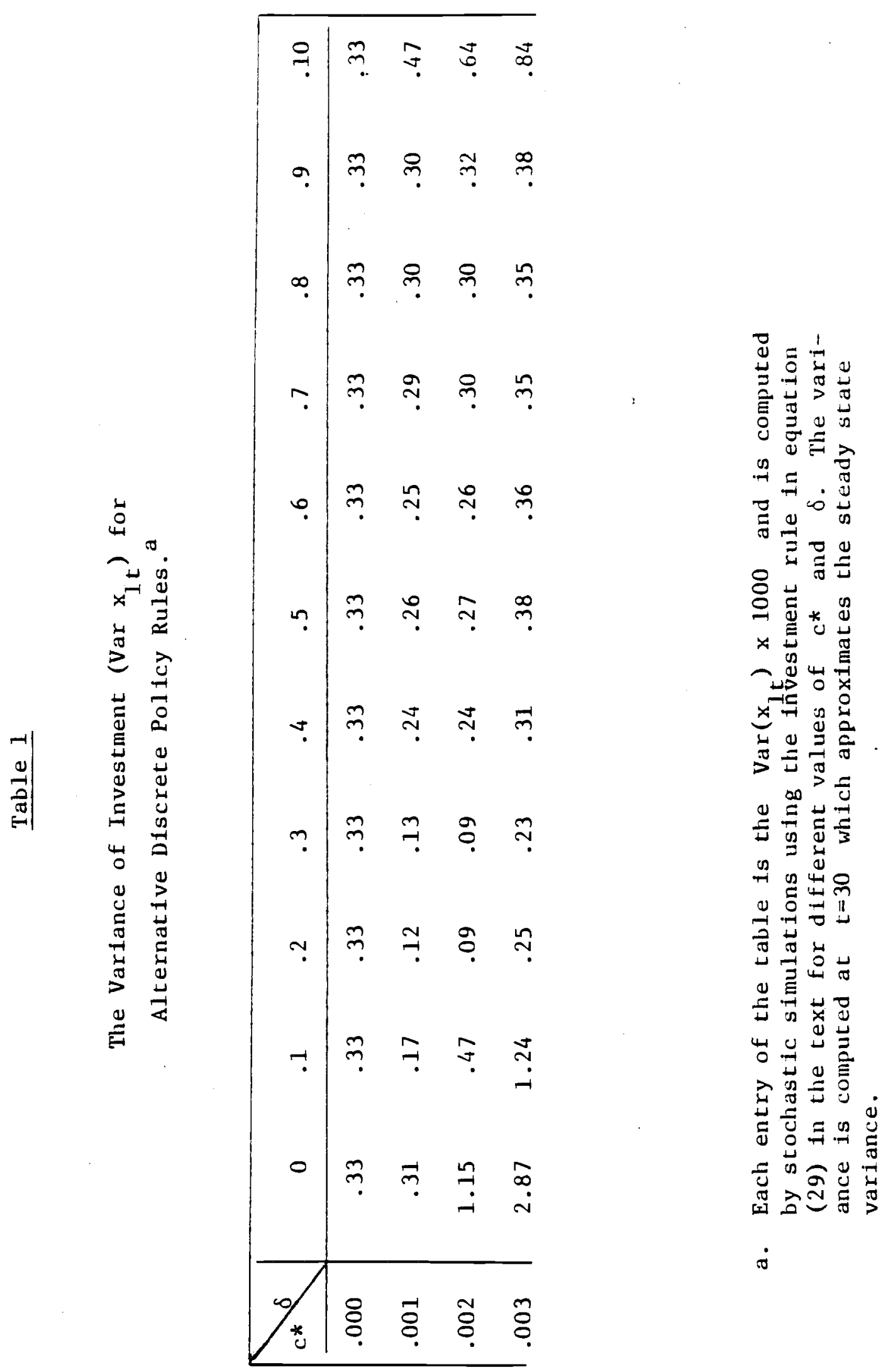


FOOTNOTES

1. See, for example, Lucas and Prescott (1971), Sargent (1979), Ch. 14, and Kydland and Prescott (1980).

2. Policy questions relating to investment in dynamic models have been addressed by Sargent (1979, p. 344), Rydland and Prescott (1980), Summers (1981), Hayashi (1982), and Taylor (1982). Lucas (1976) addresses similar policy issues in a more general setting.

3. This approach to investment demand which emphasizes heterogeneous gestation lags was applied to a Swedish investment problem in Taylor (1982) .

4. Where confusion does not arise, we generally omit a comma between the different indices in the double subscripts. No multiplication of subscript indices appears in this paper.

5. For example, in the United States the investment tax credit Jepends on the useful life of the capital equipment purchased.

6. Christiano (1982) has shown analytically that such perverse destabilization can occur when $y_{t}$ follows an $\operatorname{ARMA}(1,1)$ process. Baumol (1961) and Howrey (1966) have investigated similar problems with suboptimal policy rules in models where anticipations of future policy do not affect decisions explicitly. 
REFERENCES

Anderson, T. W. (1971), The Statistical Analysis of Time Series, John Wiley and Sons, Inc., New York.

Baumol, W. J. (1961), "Pitfalls in Contracyclical Policies: Some Tools and Results," Review of Economics and Statistics, 43, 21-26.

Box, G. E. P. and G. M. Jenkins (1970), Time Series Analysis Forecasting and Control, Holden-Day, San Francisco.

Chow, G. C. (1980), "Econometric Policy Evaluation and Optimization under Rational Expectations," Journal of Economic Dynamics and Controi, $2,47-60$.

Christiano, L. J. (1982), "Can Automatic Stabilizers be Destabilizing: An 01d Question Revisited," unpublished paper, University of Chicago.

Hayashi, F. (1982), "Tobin's Marginal q and Average q: A Neoclassical Interpretation," Econometrica, 50, 213-214.

Howrey, E. P. (1966), "Stabilization Policies in Linear Stochastic Systems, Econometric Research Program, Research Memorandum No. 83, Princeton University.

Kydland, F. and E. C. Prescott (1980), "A Competitive Theory of Fluctuations and The Feasibility and Desirability of Stabilization Policy," Rational Expectations and Economic Policy (S. Fischer, ed.), The University of Chicago Press for the National Bureau of Economic Research, Chicago.

Lucas, R. E., Jr. (1976), "Econometric Policy Evaluation: A Critique," Carnegie-Rochester Conference Series on Policy Policy, $1,19-46$.

Lucas, R. E., Jr. and E. C. Prescott (1971), "Investment under Uncertainty," Econometrica, 39, 659-681.

Sargent, T. J. (1979), Macroeconomic Theory, Academic Press, New York.

Summers, L. H. (1981), Taxation and Corporate Investment: A q-theory Approach," Brookings Papers on Economic Activity, No. 1, 67-127.

Taylor, J. B. (1982), "The Swedish Investment Funds System as a Stabilization Policy Rule," Brookings Papers on Economic Activity, No. 1 ,
57-99. 